\title{
Patients' satisfaction and subjective happiness after refractive surgery for myopia
}

This article was published in the following Dove Press journal:

Patient Preference and Adherence

\author{
Shinichiro Matsuguma' \\ Kazuno Negishi' \\ Motoko Kawashima' \\ Ikuko Toda ${ }^{2}$ \\ Masahiko Ayaki' \\ Kazuo Tsubota ${ }^{1,2}$ \\ 'Department of Ophthalmology, \\ Keio University School of Medicine, \\ Tokyo, Japan; ${ }^{2}$ Minamiaoyama Eye \\ Clinic, Tokyo, Japan
}

Purpose: The purpose of this study was to assess patients' satisfaction and change in subjective happiness after laser-assisted in situ keratomileusis (LASIK).

Patients and methods: This was a retrospective cross-sectional study of 307 patients (mean age, 34.3 years; range, 20-63 years) who underwent bilateral LASIK surgery and ocular examinations, including subjective refraction and visual acuity axial length measurements at Minamiaoyama Eye Clinic. Patients also completed questionnaires on their satisfaction with the surgery, and on the subjective happiness scale (SHS) within 1 month before and 1 month after surgery. A multiple regression analysis was performed to determine the independent predictors of the SHS score.

Results: In total, $91.2 \%$ of the patients were satisfied with surgery (very satisfied: $\mathrm{n}=155$; satisfied: $\mathrm{n}=125)$. The SHS score increased postoperatively from $5.2 \pm 0.9$ to $5.3 \pm 0.9(P<0.001)$. The multiple regression analysis revealed that the preoperative SHS score $(\beta=0.77 ; P<0.001)$ and satisfaction with surgery $(\beta=-0.11 ; P=0.05)$ were predictors of postoperative SHS score.

Conclusion: LASIK may contribute to increased patient happiness.

Keywords: satisfaction, subjective happiness, laser in situ keratomileusis, eye surgery

\section{Introduction}

Laser-assisted in situ keratomileusis (LASIK) is the most popular refractive surgery performed to correct refractive errors of the eye, including myopia, hyperopia, and astigmatism. LASIK surgery has been reported to yield significantly improved patientreported visual outcomes. ${ }^{1}$ The result is the patient's independence from glasses and contact lenses.

Many studies have reported that LASIK surgery can improve not only visual function, ${ }^{1}$ but also patients' quality of life (QOL). ${ }^{2-9}$ Patient-reported outcomes also revealed that LASIK can maximize patient satisfaction and QOL by minimizing the onset of visual and dry eye symptoms. ${ }^{10}$

Happiness or positive emotions are now regarded as a critical component of health. ${ }^{11}$ With the introduction of the discipline of positive psychology in the late 1990s, many scientific investigations of happiness have resulted in the development of the proposal that happiness or positive emotions are highly beneficial for physical and mental health. ${ }^{11-18}$

Some studies have reported an association between surgical outcomes and happiness. ${ }^{19,20}$ However, no study on the relationship between patients' satisfaction and happiness in patients who underwent ophthalmic surgery is available on PubMed or EBSCOhost; the period until May 2018 was searched using the keywords "happiness," “eye," "Patient reported outcome (PRO)," "QOL," and "subjective well-being."
Correspondence: Kazuno Negishi Department of Ophthalmology, Keio University School of Medicine, 35 Shinjuku-ku, Tokyo, 160-8582, Japan $\mathrm{Tel}+8 \mid 33353$ I2II

Fax +8I 333598302

Email fwic7788@mb.infoweb.ne.jp 
In this study, we investigated changes in patients' subjective happiness after LASIK. In addition, we analyzed the influence of patients' visual function as well as their satisfaction with the surgery on subjective happiness.

\section{Patients and methods}

The study participants were patients who underwent bilateral LASIK procedures at the Minamiaoyama Eye Clinic, Minato-ku, Tokyo, between September 2011 and August 2014. Subjects completed a questionnaire of their subjective happiness within 1 month before and 1 month after surgery, as well as a questionnaire of their satisfaction with surgery 1 month after surgery.

Subjects who did not respond to all questions were excluded. The institutional review board of the Minamiaoyama Eye Clinic approved the research protocol, and the study was conducted according to the tenets of the Declaration of Helsinki.

This study was a retrospective chart review of patients at the eye clinic. A written informed consent was obtained from all patients before LASIK surgery, and an opt-out consent method was used to participate in this study. During the study period, 1,159 patients underwent LASIK

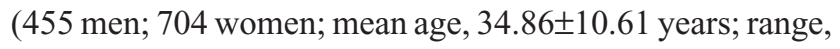
16-67 years). A total of 337 patients underwent either bilateral or unilateral surgery and completed the questionnaires before and 1 month after surgery. Of those, 307 patients who underwent bilateral LASIK and completed all questionnaires were included in this study (113 men; 194 women). The mean patient age was $34.34 \pm 9.38$ years (range, $20-63$ years). The target refraction was emmetropia in 301 patients, and in the other 6 patients, a monovision approach was applied, in which the dominant eye was treated for distance vision and the non-dominant eye for near vision. Patients using psychiatric medication were not indicated for surgery.

Bilateral LASIK procedures were performed in succession on each patient using an identical method. The corneal flap was created using a MK-2000 microkeratome (Nidek Co., Ltd., Aichi, Japan), an IntraLase FS60 (Abbott Medical Optics, Inc., Tokyo, Japan), or an IntraLase iFS laser (Abbott Medical Optics, Inc.). Laser ablation was performed using the EC-5000 CXII excimer laser (Nidek Co., Ltd.). The interface between the flap and the stromal bed was irrigated with a balanced salt solution containing $0.6 \mathrm{mg} / \mathrm{mL}$ gentamicin (Gentacin; Schering-Plough KK, Osaka, Japan) and $0.4 \mathrm{mg} / \mathrm{mL}$ methylprednisolone (Solu-Medrol; Pfizer, Inc., Tokyo, Japan). Postoperatively, a low-dose steroid (0.1\% Flumetholone ${ }^{\mathrm{TM}}$; Santen Pharmaceutical Co., Ltd.,
Osaka, Japan), antibiotics (Tarivid and Cravit ${ }^{\circledR}$; Santen Pharmaceutical Co., Ltd.), and $0.3 \%$ hyaluronic acid eyedrops (Hyalein ${ }^{\circledR}$; Santen Pharmaceutical Co., Ltd.) were used 5 times daily and discontinued 1 week postoperatively.

Experienced ophthalmic examiners performed pre- and postoperative ocular examinations, including measurement of the uncorrected distance visual acuity (UDVA), manifest refraction (spherical equivalent of subjective manifest refraction [SE]), and spherical (S) and cylindrical powers (C).

Regarding outcome measures, subjective happiness was measured using the validated Japanese version of the subjective happiness scale (SHS) ${ }^{21}$ originally developed by Lyubomirsky and Lepper. ${ }^{22}$ The scale is a 4-item questionnaire of subjective global happiness; each item requires patients to rate the statements on a 7-point Likert scale. The possible scores range from 1 to 7 , and higher values corresponded to higher subjective happiness. The overall SHS score was calculated by taking the mean of the responses to the 4 items. Both internal consistency ( $\alpha=0.80$ for males; $\alpha=0.84$ for females) and test-retest reliability ( $r=0.86)$ over 5 weeks were scientifically sound (Table 1$){ }^{22}$

In addition, a 1-item questionnaire rated on a 4-point Likert scale ranging from 1 (very satisfied) to 4 (least satisfied) was used to measure patient satisfaction with surgery. A lower score indicated a higher level of satisfaction. Since there was no difference between the averaged visual acuity of the 2 eyes and that of the right eye, the data obtained from the right eyes of the included patients were used for all statistical analyses. Visual acuity was converted to the logarithm of the minimum angle of resolution, and the Snellen equivalent was also provided. We first conducted a paired $t$-test to determine the differences in the UDVA, SE, S, and C powers before and 1 month after surgery. Differences between the

Table I Subjective happiness scale (SHS)

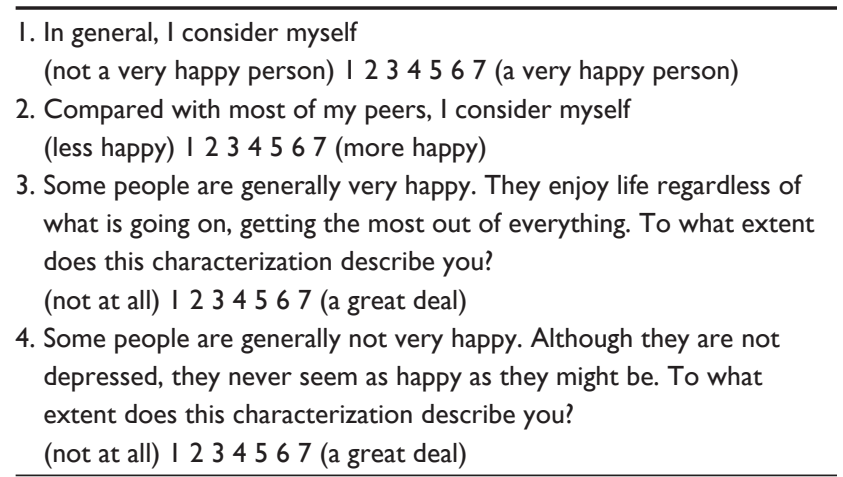

Notes: To score the scale, the fourth item was reverse-coded (ie, $7=1,6=2$, etc.) and the means were summed up. Adapted by permission from Springer Nature: Springer Nature. Soc Indic Res. 46(2):I37-155. A measure of subjective happiness: preliminary reliability and construct validation. Lyubomirsky S, Lepper HS. 1999.22 
pre- and postoperative SHS scores were tested using the Wilcoxon signed-rank test. We then performed a multiple regression analysis to estimate the factors affecting patient satisfaction with LASIK surgery. We also used the KruskalWallis and Tukey post hoc tests to investigate the group comparison associations between patient satisfaction with surgery and postoperative UDVA. Finally, we conducted a multiple regression analysis to investigate the predictors of the postoperative SHS score on the possible predictor variables (preoperative SHS score, postoperative UDVA, sex, and age). A $P$-value $<0.05$ was considered significant. All statistical analyses were performed using SPSS version 24 for Windows (SPSS Inc., Chicago, IL, USA).

\section{Results}

\section{Results of ocular examinations and questionnaires}

During the study period, 1,159 patients underwent LASIK; 337 underwent either bilateral or unilateral surgery and completed the pre- and postoperative questionnaires. This study analyzed 307 patients (113 men; 194 women) who underwent bilateral LASIK and completed all questionnaires. The mean patient age was $34.3 \pm 9.4$ years (range, 20-63 years). The pre- and postoperative results of each variable are shown in Table 2. The mean score of the satisfaction with surgery was very high; $50.5 \%$ of the patients were very satisfied with surgery and $40.7 \%$ were satisfied. We also analyzed only the standard procedure cases $(n=301)$, but the result of each

Table 2 Results of ocular examinations and questionnaires

\begin{tabular}{|c|c|c|c|}
\hline Variables & $\begin{array}{l}\text { Preoperative } \\
\text { mean } \pm \text { SD } \\
\text { (range) }\end{array}$ & $\begin{array}{l}\text { Postoperative } \\
\text { mean } \pm \text { SD } \\
\text { (range) }\end{array}$ & $P$-value \\
\hline SHS score & $\begin{array}{l}5.20 \pm 0.92 \\
(2.75-7.00)\end{array}$ & $\begin{array}{l}5.34 \pm 0.93 \\
(2.00-7.00)\end{array}$ & $<0.00 I^{a}$ \\
\hline UDVA (logMAR) & $\begin{array}{l}1.15 \pm 0.31 \\
(0.00-1.70)\end{array}$ & $\begin{array}{l}-0.10 \pm 0.15 \\
(-0.30-0.70)\end{array}$ & $<0.00 I^{\mathrm{a}}$ \\
\hline SE (diopters) & $\begin{array}{l}-2.91 \pm 1.20 \\
(-6.25-0.88)\end{array}$ & $\begin{array}{l}-0.04 \pm 0.25 \\
(-1.25-0.88)\end{array}$ & $<0.00 \mathrm{I}^{\mathrm{a}}$ \\
\hline S (diopters) & $\begin{array}{l}-4.98 \pm 2.29 \\
(-11.50-2.25)\end{array}$ & $\begin{array}{l}0.04 \pm 0.41 \\
(-2.50-1.75)\end{array}$ & $<0.00 I^{a}$ \\
\hline C (diopters) & $\begin{array}{l}-0.84 \pm 0.74 \\
(-3.75-0.00)\end{array}$ & $\begin{array}{l}-0.12 \pm 0.30 \\
(-2.50-0.00)\end{array}$ & $<0.00 \mathrm{I}^{\mathrm{a}}$ \\
\hline Satisfaction with surgery & - & $\begin{array}{l}1.59 \pm 0.67 \\
(1.00-4.00)\end{array}$ & - \\
\hline
\end{tabular}

Notes: The paired $t$-test: difference between the pre- and postoperative UDVA, SE, and $S$ and $C$ powers. The Wilcoxon signed-rank test: difference between the preand postoperative SHS scores. ${ }^{a} P<0.05$. "-" indicates data not applicable.

Abbreviations: SHS, subjective happiness scale; UDVA, uncorrected distance visual acuity; SE, spherical equivalent of subjective manifest refraction; S, spherical power; C, cylindrical power.
Table 3 The predictors in multiple regression analysis on the factors to identify factors associated with the satisfaction with surgery

\begin{tabular}{lll}
\hline Independent variables & $\boldsymbol{\beta}$ & $\boldsymbol{P}$-value \\
\hline Preoperative SHS & $-0.17^{\mathrm{a}}$ & $<0.001$ \\
Preoperative UDVA & -0.05 & 0.35 \\
Postoperative UDVA & $0.40^{\mathrm{a}}$ & $<0.001$ \\
Sex & -0.05 & 0.36 \\
Age & $0.15^{\mathrm{a}}$ & 0.01 \\
\hline
\end{tabular}

Notes: Dependent variable: satisfaction with surgery. ${ }^{\mathrm{P}}<0.05$.

Abbreviations: SHS, subjective happiness scale; UDVA, uncorrected distance visual acuity; $\beta$, standardized beta coefficients.

difference between pre- and postoperative variables and the result of the following analyses were similar.

\section{Factors affecting satisfaction with surgery}

Multiple regression analysis revealed that postoperative UDVA $(\beta=0.40 ; P<0.001)$, the preoperative SHS score $(\beta=-0.17 ; P<0.001)$, and age $(\beta=0.15 ; P=0.01)$ were the significant factors that affected patients' satisfaction with surgery (Table 3 ).

The correlation between postoperative UDVA and satisfaction with surgery is shown in Figure 1. The KruskalWallis test revealed a significant difference in postoperative UDVA according to the satisfaction score, and the Tukey

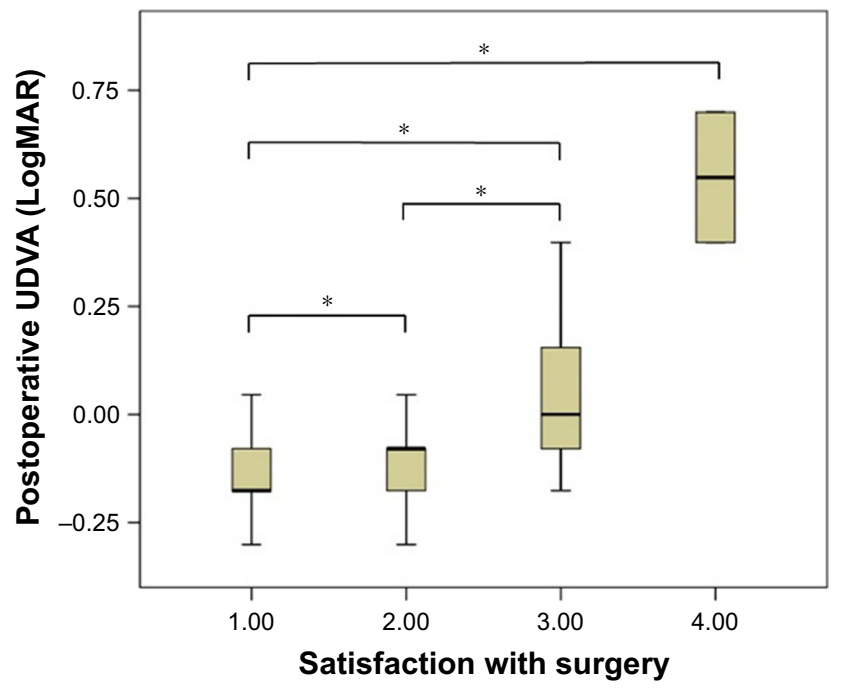

Figure I Association between postoperative UDVA and satisfaction with surgery. Notes: A lower score indicates better UDVA. Satisfaction with surgery: A lower score indicates a higher level of satisfaction with LASIK surgery: $1.00 \quad(n=155)$, $2.00(n=125), 3.00(n=25), 4.00(n=2)$. $* P<0.00$ I. Box plots showing the distribution of postoperative UDVA. It should be noted that the group of patients with higher satisfaction with their surgery had significantly better UDVA compared with the other groups with lower satisfaction with surgery. ( ${ }^{*} P<0.01$, Tukey post hoc test). The horizontal line in each diagram indicates the median scores of their UDVA. The height, positive error bar, and negative error bar of each box indicate the 25-75th percentiles, maximum values, and minimum values, respectively.

Abbreviations: UDVA, uncorrected distance visual acuity; LASIK, laser-assisted in situ keratomileusis. 
Table 4 The predictors in multiple regression analysis on the factors to identify factors associated with postoperative subjective happiness

\begin{tabular}{lll}
\hline Independent variables & $\boldsymbol{\beta}$ & $\boldsymbol{P}$-value \\
\hline Preoperative SHS & $0.77^{\mathrm{a}}$ & $<0.00 \mathrm{I}$ \\
Postoperative UDVA & 0.05 & 0.88 \\
Satisfaction with surgery & $-0.1^{\mathrm{a}}$ & 0.05 \\
Sex & -0.06 & 0.39 \\
Age & 0.00 & 0.33 \\
\hline
\end{tabular}

Notes: Dependent variable: postoperative SHS. ${ }^{a}<<0.05$.

Abbreviations: SHS, subjective happiness scale; UDVA, uncorrected distance visual acuity; $\beta$, standardized beta coefficients.

post hoc test revealed that between-group differences existed $(P<0.01)$.

\section{Predictors of postoperative subjective happiness}

The multiple regression analysis of variables, including postoperative UDVA, preoperative SHS, age, sex, and satisfaction with surgery demonstrated that the preoperative SHS score $(\beta=0.77, P<0.001)$ and satisfaction with surgery ( $\beta=-0.11, P=0.05)$ were predictors of the postoperative SHS score (Table 4).

\section{Discussion}

To the best of our knowledge, this is the first study in ophthalmology to evaluate the effect of surgical treatment on patients' subjective happiness, as well as the relationship between patients' satisfaction with surgery and subjective happiness. We found that LASIK cannot only achieve high satisfaction with surgery, as reported previously, ${ }^{9}$ but also can improve patients' subjective happiness postoperatively. In addition, postoperative subjective happiness was influenced by preoperative subjective happiness and satisfaction with surgery.

There have been several previous reports on happiness and ocular disease, ${ }^{10,19,20,23,24}$ though most of the studies used non-validated questionnaires. Cumberland et al evaluated the frequency and distribution of laser refractive surgery in the UK and the outcomes of happiness in a cross-sectional study, and reported that there was no difference in self-reported happiness between those who underwent the surgery and who did not. ${ }^{23}$ Bullock et al examined other perceived personal characteristics, such as intelligence, friendliness, trustworthiness, and happiness among those who underwent surgery for blepharoptosis and dermatochalasis, and reported that perceived postoperative happiness increased. ${ }^{20}$ Kawashima et al examined the subjective happiness of office workers with subjective and/or objective dry eye symptoms with the validated SHS questionnaire used in this study. ${ }^{10}$ According to that study, the mean SHS score among patients with subjective and objective dry eye symptoms was $4.87 \pm 1.00 .{ }^{10}$ Additionally, the mean SHS score among the sample of healthy Japanese college students was $4.68 \pm 1.05 .{ }^{21}$ The baseline mean SHS score of patients who underwent LASIK surgery was relatively higher than that of patients with dry eye $^{10}$ or healthy college students. ${ }^{21}$ Several socioeconomic factors, such as income may affect subjective happiness. ${ }^{12}$ The higher SHS scores of LASIK patients may be attributable to differences in the social background of those patients. Those who can access LASIK surgery already have some privilege, and the relatively higher happiness may be due to increased income. Additionally, laser treatment was more common among those who participated more frequently in social activities and attended sports/gym clubs. ${ }^{23}$ As social relationships and exercise are associated with happiness, ${ }^{12}$ the mean baseline SHS among the LASIK patients might be higher for that reason.

QOL is the most important concept in patient-reported outcome measures. Several studies have reported improvements in the QOL of patients after LASIK. ${ }^{6-9}$ Lazon de la Jara et al reported improvement in the QOL of myopia patients after LASIK using the Institute for Eye Research multidimensional QOL scale for myopia. ${ }^{7}$ Their research confirmed that LASIK improved the patients' adaptability, confidence, and sense of well-being, allowing them to have a more optimistic attitude about their lives and increased perceived QOL after surgery. Sandoval et al conducted a systematic review of QOL after LASIK surgery and concluded that recent LASIK surgery can improve the QOL. ${ }^{9}$ These reports suggested that LASIK positively affects the patient's psychological profile, resulting in increased postoperative happiness.

Several reports in various fields have addressed the association between the QOL and subjective happiness. Kang et al identified the association between QOL and happiness among cancer survivors. ${ }^{25}$ Hirosaki et al reported a correlation between subjective happiness and QOL in community-dwelling elderly Japanese using a $100 \mathrm{~mm}$ visual analog scale. ${ }^{26}$ In dentistry, Tuchtenhagen et al reported that the oral health-related QOL in schoolchildren was closely associated with the SHS score. ${ }^{27}$ These reports may support our findings that improved QOL after LASIK was linked to subjective happiness.

We also found that age, postoperative UDVA, and the preoperative SHS score were significantly associated with patient satisfaction with surgery. In addition, the patients 
with higher satisfaction had better postoperative UDVA. Satisfaction with surgery and the preoperative SHS score were also significant predictors of the postoperative SHS score. As postoperative UDVA is related to the QOL, the improvement in QOL would result in higher satisfaction with LASIK surgery. ${ }^{6-9}$ When considered together, we speculate that LASIK surgery could contribute to patients' subjective happiness by improving the QOL.

Interestingly, the preoperative SHS score was an important predictor of both satisfaction with surgery and the postoperative SHS score. This result confirms that happy people tend to perceive life events in more positive ways compared with unhappy one. ${ }^{28}$ Considering these traits of happy people, it is understandable that satisfaction with surgery and improvements in the SHS score were higher among the patients with higher preoperative SHS scores.

Regarding age, this study revealed that age was negatively associated with the satisfaction with surgery. This result was in line with that of the previous studies, which indicated that older age is an important risk factor for a decrease in satisfaction with LASIK surgery. ${ }^{?}$

Many previous studies have reported a correlation between happiness and physical health. For example, happy people have lower levels of inflammation, ${ }^{13}$ lower cortisol levels, ${ }^{15}$ and better recovery of the cardiovascular system. ${ }^{16}$ Happy people also tend to have better lifestyle habits that include a proper diet and moderate exercise. ${ }^{17}$ Thus, some longitudinal studies have indicated that happiness is a causal factor of longevity. ${ }^{11,17}$ Some research has also indicated that happiness is a causal factor in the achievement of higher incomes and financial success later in life. ${ }^{12}$ In this aspect, LASIK might become a promising contributor to the patient's happiness.

A limitation of this study was that we could not collect additional data that might affect subjective happiness, such as income or marital status, owing to the retrospective nature of the study. ${ }^{12}$ However, it is reasonable to consider that no dramatic changes in these variables occurred in 1 month. Volunteer bias might be a factor due to the nature of this study. Finally, the patients' level of happiness might change over time, considering the set-point theory of happiness. ${ }^{29}$ Additional studies should be conducted to confirm our results over longer periods of time, such as 3 months or 1 year.

\section{Conclusion}

The results of the present study showed that LASIK positively affected patients' overall subjective happiness postoperatively, and this improvement in subjective happiness was correlated with satisfaction with surgery, which could be increased by the increased QOL after LASIK surgery.

\section{Acknowledgment}

We are very grateful to Tomoo Oobayashi of Minamiaoyama Eye Clinic for helping us in the collection of data.

\section{Disclosure}

The author reports no conflicts of interest in this work.

\section{References}

1. Moshirfar M, Shah TJ, Skanchy DF, Linn SH, Durrie DS. Meta-analysis of the FDA Reports on Patient-Reported Outcomes Using the Three Latest Platforms for LASIK. J Refract Surg. 2017;33(6):362-368.

2. Spaeth G, Walt J, Keener J. Evaluation of quality of life for patients with glaucoma. Am J Ophthalmol. 2006;141(1 Suppl):S3-S14.

3. Mangione CM, Lee PP, Pitts J, Gutierrez P, Berry S, Hays RD. Psychometric properties of the National Eye Institute Visual Function Questionnaire (NEI-VFQ). NEI-VFQ Field Test Investigators. Arch Ophthalmol. 1998;116(11):1496-1504.

4. Mangione CM, Lee PP, Gutierrez PR, et al. Development of the 25-item National Eye Institute Visual Function Questionnaire. Arch Ophthalmol. 2001;119(7):1050-1058.

5. Suzukamo Y, Oshika T, Yuzawa M, et al. Psychometric properties of the 25-item National Eye Institute Visual Function Questionnaire (NEI VFQ-25), Japanese version. Health Qual Life Outcomes. 2005;3:65.

6. Hays RD, Tarver ME, Spritzer KL, et al. Assessment of the Psychometric Properties of a Questionnaire Assessing Patient-Reported Outcomes With Laser In Situ Keratomileusis (PROWL). JAMA Ophthalmol. 2017; 135(1):3-12.

7. Lazon de La Jara P, Erickson D, Erickson P, Stapleton F. Visual and non-visual factors associated with patient satisfaction and quality of life in LASIK. Eye. 2011;25(9):1194-1201.

8. Solomon KD, Fernández de Castro LE, Sandoval HP, et al. LASIK world literature review: quality of life and patient satisfaction. $O p h$ thalmology. 2009;116(4):691-701.

9. Sandoval HP, Donnenfeld ED, Kohnen T, et al. Modern laser in situ keratomileusis outcomes. J Cataract Refract Surg. 2016;42(8): 1224-1234.

10. Kawashima M, Uchino M, Yokoi N, et al. Associations between subjective happiness and dry eye disease: a new perspective from the Osaka study. PLoS One. 2015;10(4):e0123299.

11. Frey BS. Psychology. Happy people live longer. Science. 2011;331(6017): $542-543$.

12. Lyubomirsky S, King L, Diener E. The benefits of frequent positive affect: does happiness lead to success? Psychol Bull. 2005;131(6): 803-855.

13. Segerstrom SC, Sephton SE. Optimistic expectancies and cell-mediated immunity: the role of positive affect. Psychol Sci. 2010;21(3): 448-455.

14. Matsunaga M, Isowa T, Yamakawa K, et al. Association between perceived happiness levels and peripheral circulating pro-inflammatory cytokine levels in middle-aged adults in Japan. Neuro Endocrinol Lett. 2011;32(4):458-463.

15. Steptoe A, Dockray S, Wardle J. Positive affect and psychobiological processes relevant to health. J Pers. 2009;77(6):1747-1776.

16. Fredrikson M, Blumenthal JA. Serum lipids, neuroendocrine and cardiovascular responses to stress in healthy Type A men. Biol Psychol. 1992; 34(1):45-58. 
17. Shirai K, Iso H, Ohira T, et al. Perceived level of life enjoyment and risks of cardiovascular disease incidence and mortality: the Japan public health center-based study. Circulation. 2009;120(11):956-963.

18. Danner DD, Snowdon DA, Friesen WV. Positive emotions in early life and longevity: findings from the nun study. J Pers Soc Psychol. 2001; 80(5):804-813.

19. Shin JH, Kim YD, Woo KI. Korean Society of Ophthalmic Plastic and Reconstructive Surgery (KSOPRS). Impact of epiphora on visionrelated quality of life. BMC Ophthalmol. 2015;15:6.

20. Bullock JD, Warwar RE, Bienenfeld DG, Marciniszyn SL, Markert RJ. Psychosocial implications of blepharoptosis and dermatochalasis. Trans Am Ophthalmol Soc. 2001;99(71):65-71.

21. Shimai S, Otake K, Utsuki N, Ikemi A, Lyubomirsky S. Development of a Japanese version of the Subjective Happiness Scale (SHS), and examination of its validity and reliability. Nihon Koshu Eisei Zasshi. 2004;51(10):845-853.

22. Lyubomirsky S, Lepper HS. A measure of subjective happiness: preliminary reliability and construct validation. Soc Indic Res. 1999;46(2): $137-155$.

23. Cumberland PM, Chianca A, Rahi JS; UK Biobank Eyes \& Vision Consortium. Laser refractive surgery in the UK Biobank study: Frequency, distribution by sociodemographic factors, and general health, happiness, and social participation outcomes. J Cataract Refract Surg. 2015;41(11):2466-2475.
24. Schallhorn SC, Venter JA, Teenan D, et al. Patient-reported outcomes 5 years after laser in situ keratomileusis. J Cataract Refract Surg. 2016;42(6):879-889.

25. Kang D, Kim IR, Choi EK, et al. Who are happy survivors? Physical, psychosocial, and spiritual factors associated with happiness of breast cancer survivors during the transition from cancer patient to survivor. Psychooncology. 2017;26(11):1922-1928.

26. Hirosaki M, Ishimoto Y, Kasahara Y, et al. Self-rated happiness is associated with functional ability, mood, quality of life and income, but not with medical condition in community-dwelling elderly in Japan. Geriatr Gerontol Int. 2011;11(4):531-533.

27. Tuchtenhagen S, Bresolin CR, Tomazoni F, et al. The influence of normative and subjective oral health status on schoolchildren's happiness. BMC Oral Health. 2015;15:15.

28. Lyubomirsky S. Why are some people happier than others? The role of cognitive and motivational processes in well-being. Am Psychol. 2001;56(3):239-249.

29. Brickman P, Coates D, Janoff-Bulman R. Lottery winners and accident victims: is happiness relative? J Pers Soc Psychol. 1978;36(8): 917-927.
Patient Preference and Adherence

\section{Publish your work in this journal}

Patient Preference and Adherence is an international, peer-reviewed, open access journal that focuses on the growing importance of patient preference and adherence throughout the therapeutic continuum. Patient satisfaction, acceptability, quality of life, compliance, persistence and their role in developing new therapeutic modalities and compounds to optimize

\section{Dovepress}

clinical outcomes for existing disease states are major areas of interest for the journal. This journal has been accepted for indexing on PubMed Central. The manuscript management system is completely online and includes a very quick and fair peer-review system, which is all easy to use. Visit http://www. dovepress.com/testimonials.php to read real quotes from published authors. 